01,03,04

\title{
Когерентная обменная корреляция в квантовых системах
}

\author{
(ㄷ С.В. Ганцевич, В.Л. Гуревич \\ Физико-технический институт им. А.Ф. Иофрфе РАН, \\ Санкт-Петербург, Россия \\ E-mail: sergei.elur@mail.ioffe.ru
}

(Поступила в Редакцию 13 июня 2017 г.)

Обсуждается с единой точки зрения ряд обменных корреляционных эффектов в квантовых системах. Эти эффекты могут наблюдаться как на микроскопических, так и на макроскопических расстояниях. Для анализа макроскопических корреляционных эффектов требуется понимание физического механизма обменной корреляции. А это, в свою очередь, требует анализа фазовых соотношений между волновыми функциями квантовых состояний, определяющих наблюдаемые физические величины. Неучет этих фазовых соотношений при интерпретации корреляционных экспериментов вызвал появление таких предположений как мгновенное дальнодействие на расстоянии и других подобных идей, противоречащих традиционной физической картине близкодействия, причинности и локальности. Наоборот, понимание физической природы корреляционного механизма позволяет объяснить эти эксперименты безо всяких такого рода идей.

DOI: $10.21883 /$ FTT.2018.01.45281.187

\section{1. Введение}

В работе дан обзор ряда обменных корреляционных эффектов с единой точки зрения в свете физических представлений, развитых в работе авторов [1]. Мы ограничимся рассмотрением системы невзаимодействующих квантовых частиц. Под корреляцией будем понимать связь между измеренными физическими величинами, которые относятся к этим частицам. Корреляцию мы будем отличать от взаимодействия. Взаимодействие обычно предполагает наличие причинно-следственной связи между двумя событиями, т.е. одно событие является причиной, другое - следствием. Для двух коррелированных событий это не обязательно. Два коррелированных события могут быть следствием некоего третьего события - общей причины в прошлом. При этом между ними нет непосредственной причинно-следственной связи.

Далее речь пойдет о корреляции измерений наблюдаемых величин в один момент времени в двух точках пространства. Взаимодействие между такими событиями невозможно, если принять положение теории относительности об отсутствии сверхсветовых скоростей.

Под наблюдаемыми физическими величинами будем понимать все, что может быть измерено прямо или косвенно. Например, это могут быть энергии, скорости, заряды, спины, положения в пространстве, а также какие-либо характеристики твердотельного устройства.

Системой можно считать уже две частицы. Простой двухчастичной квантовой системой является, например, два электрона в атоме гелия или в молекуле водорода. Здесь нам достаточно рассмотреть только двухчастичные корреляции, поскольку многочастичные корреляции могут быть построены на основе и по образцу двухчастичных.

Корреляция между классическими частицами возможна только в результате взаимодействия между ними.
А вот для квантовых частиц это не обязательно. Корреляция между наблюдаемыми физическими величинами, которые определяются состояниями квантовых частиц, может возникать и безо всякого явного взаимодействия между частицами. Это так называемая обменная корреляция, о которой будет идти речь.

В последние десятилетия чрезвычайно популярна квантовая корреляция на больших макроскопических расстояниях между парой измерений. Общее название всего этого - парадокс Эйнштейна-ПодольскогоРозена по имени авторов работы 1935 года [2], где впервые было указано на видимое противоречие между предписаниями квантовой механики и теорией относительности.

В настоящее время существует огромная литература по корреляции измерений на макроскопических расстояниях (см., например, [3-7], а также [8-13]. Несмотря на такое изобилие, до сих пор нет общепринятой бесспорной точки зрения на этот по виду загадочный эффект. Его трудно объяснить, принимая одновременно квантовую механику и теорию относительности. Действительно, прямая связь между событиями в один момент времени невозможна ввиду конечности скорости света, а из принципов квантовой механики следует, что значения наблюдаемых величин создаются в момент измерения и до измерения не существуют.

Таким образом, обе возможные причины для существования корреляции измерений, как будто, отсутствуют. Но корреляция, тем не менее, наблюдается. Именно это обстоятельство и порождает нескончаемый поток литературы по квантовой корреляции на макроскопических расстояниях с самыми разнообразными объяснениями. Более того, это явление, как странное и таинственное, постоянно упоминается в научной и научно-популярной литературе, в частности, в научных программах радио и телевидения. 
Далее мы покажем, что физическая природа корреляции одна и та же для микроскопических и макроскопических расстояний. Сходство между различными проявлениями одного и того же физического явления позволяет лучше понять его механизм. Оказывается, что физическая причина наблюдаемой корреляции - фазовые соотношения между волновыми функциями квантовых частиц, определяюших наблюдаемые физические величины и участвующих в квантовом обмене. При общепринятом подходе к изучению квантовой корреляции до сих пор не обращали внимания на эти соотношения.

\section{2. Постоянные и переменные наблюдаемые величины}

Своеобразие квантовой механики состоит в том, что уравнение Шредингера, которое определяет волновую функцию - линейно и допускает суперпозицию решений, в то время как наблюдаемые физические величины определяются билинейной комбинацией волновой функции и ее комплексного сопряжения. Это важное обстоятельство приводит к появлению двух существенно разных вкладов в наблюдаемые величины, которые условно можно назвать фоновым вкладом и флуктуационным вкладом. Покажем, как появляются эти два вклада и в чем заключается их различие.

Следуя Дираку, для краткости и удобства будем называть волновую функцию $\psi \equiv|\psi\rangle-k e t$-функцией, или просто ket, а комплексно-сопряженную функцию

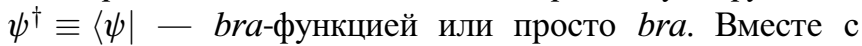
оператором наблюдаемой величины - оператором $\hat{c}$, эти bra и ket составляют скобку Дирака - bracket, которая и дает среднее значение наблюдаемой физической величины. Рассмотрим простой случай одночастичных величин и точечного оператора $\hat{c} \equiv C(r)$. (Здесь и далее мы опускаем векторные значки.) Наблюдаемая величина $\bar{C}$ равна

$$
\bar{C}=\langle\psi|C(r)| \psi\rangle \equiv \int_{V} d r \psi^{\dagger}(r, t) C(r) \psi(r, t) .
$$

Пусть, например, $C(r)=\delta(r-R)$. Из формулы (1) следует:

$$
\bar{C}=\langle\psi \mid \psi\rangle \equiv \int_{V} d r \psi^{\dagger}(r, t) \delta(r-R) \psi(r, t)=|\psi(R, t)|^{2}
$$

Рассмотрим теперь две собственные функции гамильтониана $H$, который не зависит от времени. Имеем

$$
H \psi_{k}=\epsilon_{k} \psi_{k} \quad \text { и } \quad H \psi_{l}=\epsilon_{l} \psi_{l} .
$$

Форма собственных функций в пространстве с течением времени сохраняется, меняется только фазовый множитель, зависящий от энергии

$$
\psi_{k}(r, t)=\psi_{k}(r) e^{-i \epsilon_{k} t} \quad \text { и } \quad \psi_{l}(r, t)=\psi_{l}(r) e^{-i \epsilon_{l} t}
$$

Составим из функций (4) линейную комбинацию

$$
\psi(r, t)=c_{k} \psi_{k}(r) e^{-i \epsilon_{k} t}+c_{l} \psi_{l}(r) e^{-i \epsilon_{l} t}
$$

и найдем с ее помощью среднее значение оператора положения $W(R)=\delta(r-R)$. Имеем:

$$
\begin{aligned}
W(R, t)= & \left|c_{k}\right|^{2}\left|\psi_{k}(R)\right|^{2}+\left|c_{l}\right|^{2}\left|\psi_{l}(R)\right|^{2} \\
& +2 \operatorname{Re}\left[c_{k}^{\dagger} c_{l} e^{i\left(\epsilon_{k}-\epsilon_{k}\right) t} \psi_{k}^{\dagger}(R) \psi_{l}(R)\right] .
\end{aligned}
$$

Интегрируя это выражение по всему пространству, получим

$$
\langle\psi \mid \psi\rangle=\left|c_{k}\right|^{2}+\left|c_{l}\right|^{2}=F_{k}+F_{l}=N .
$$

Здесь $F_{k}$ и $F_{l}$ - числа заполнения состояний $k$ и $l$, а $N-$ полное число частиц в этих состояниях. Из (7) следует, что $c_{k}=e^{i \varphi_{k}} \sqrt{F_{k}}$ и $c_{l}=e^{i \varphi_{l}} \sqrt{F_{l}}$. При этом начальные фазы $\varphi_{k}$ и $\varphi_{l}$ никак не влияют на числа заполнения. Если считать эти начальные фазы произвольными случайными величинами и усреднить по ним произведение (6), то останутся только состояния с одинаковыми индексами у бра и кет функций. Тот же результат получится также при усреднении по времени, если частоты $\epsilon_{k}$ и $\epsilon_{l}$ не совпадают.

$$
\overline{W(R)}=\left|\psi_{k}(R)\right|^{2} F_{k}+\left|\psi_{l}(R)\right|^{2} F_{l} .
$$

Итак, мы видим, что принцип суперпозиции вместе с квадратичностью выражений для наблюдаемых физических величин приводит к появлению двух различных вкладов в наблюдаемые физические величины. За один вклад ответственны пары бра + кет собственных функций гамильтониана с одинаковыми квантовыми индексами. Этот вклад не зависит ни от времени, ни от начальных фаз волновых функций. Состояния $(k k)$ и $(l l)$, обеспечивающие такой вклад, можно назвать чистыми квантовыми состояниями или чистыми бра + кет парами. Вклад от чистых пар создает постоянный фон значений наблюдаемых физических величин.

Второй вклад в наблюдаемые величины дают пары бра+кет с различными квантовыми индексами. (В формуле (6) это $(k l)$ и $(l k)$.) Такие пары можно назвать смешанными бра + кет парами или смешанными квантовыми состояниями. Вклад смешанных пар зависит от разности фаз бра и кет функций в момент наблюдения: $\pm \phi(t)=\left(\epsilon_{k}-\epsilon_{l}\right) t+\varphi_{k}-\varphi_{l}$. Зависимость наблюдаемых величин от фаз $\phi(t)$ приводит к тому, что этот вклад исчезает при усреднении по времени или по ансамблю. Таким образом, вклад от смешанных состояний представляет собой знакопеременные флуктуации над постоянным фоном от чистых состояний. Именно это приводит к отсутствию их вклада в выражениях для одночастичных наблюдаемых, где усреднение по ансамблю всегда неявно предполагается.

Другим важным свойством смешанных бра + кет пар является то, что они возникают из имеющихся чистых 
пар с числами заполнения не поодиночке, но в виде двух коррелированных по фазе комбинаций. В этих комбинациях две коррелированные бра +кет пары имеют одинаковую по величине, но противоположную по знаку фазу $\phi$. Взятые поодиночке смешанные бра + кет пары исчезают при усреднении по фазе, но произведение двух таких пар с равными и противополжными фазами уже от фаз не зависит и сохраняется при усреднении. Две такие смешанные бра + кет пары можно сопоставить с постоянно упоминаемыми в литературе так называемыми запутанными состояниями (entangled states). При наличии хотя бы двух занятых состояний коррелированные по фазе бра + кет пары должны с неизбежностью возникать путем квантового обмена и давать соответствующий вклад в корреляцию наблюдаемых физических величин.

Следует заметить, что смешанное состояние возникает также при действии возмущения на чистое состояние. Поскольку возмущающий потенциал может действовать как на бра, так и на кет, то в первом приближении по возмущению также возникают два коррелированных смешанных состояния.

\section{3. Квантовый обмен}

Квантовый обмен - давно и хорошо известное в многочастичных системах явление. Впервые это понятие возникло при построении многочастичной волновой функции электронов атома. Первоначальным приближением для такой функции было приближение Хартри, когда многоэлектронная волновая функция берется в виде простого произведения одноэлектронных волновых функций.

В приближении Фока многоэлектронная волновая функция берется в виде определителя из этих же функций с переставленными координатами электронов. Для двух электронов и двух квантовых состояний $u$ и $v$ определитель Фока равен

$$
\Psi\left(r, r^{\prime}\right)=\psi_{k}(r) \psi_{l}\left(r^{\prime}\right)-\psi_{k}\left(r^{\prime}\right) \psi_{l}(r) .
$$

Это выражение есть суперпозиция двух произведений Хартри, отличающихся перестановкой координат или же квантовых индексов.

Появление двух слагаемых в формуле (9) и билинейность выражений для наблюдаемых величин приводит к появлению квантового обмена. Следует подчеркнуть, что различие между обычным и обменным вкладом проявляется только тогда, когда вычисляются наблюдаемые физические величины. На уровне волновых функций оба слагаемых в формуле (9) равноправны. Смешанные состояния с разностью фаз $k$ и $l$ функций возникают только при перемножении $\Psi^{\dagger}$ и $\Psi$, подставленных в матричный элемент наблюдаемой величины.

Кроме того, для проявления обменного вклада нужен двухчастичный оператор, который описывает две наблюдаемые величины. Для одной величины приближение Фока и приближение Хартри дают одинаковые результаты для ее значений.
Найдем такие значения для одночастичных операторов $A$ и $B$. Оператор $A$ действует на координаты $r$, а оператор $B$ на координаты $r^{\prime}$. Тогда матричные элементы этих операторов на волновой функции (9) равны

$$
\left\langle\Psi\left(r, r^{\prime}\right)|A| \Psi\left(r, r^{\prime}\right)\right\rangle=\langle k|A| k\rangle+\langle l|A| l\rangle
$$

и аналогично для оператора $B$ :

$$
\left\langle\Psi\left(r, r^{\prime}\right)|B| \Psi\left(r, r^{\prime}\right)\right\rangle=\langle k|B| k\rangle+\langle l|B| l\rangle .
$$

При выводе этих формул мы учли, что одночастичные волновые функции ортогональны и нормированы $\langle k \mid l\rangle=\langle l \mid k\rangle=0$ и $\langle k \mid k\rangle=\langle l \mid l\rangle=1$.

Как видно из (10) и (11), несмотря на исходную двухчастичную волновую функцию (9), получилась простая сумма вкладов от двух одночастичных состояний. Единственным следствием формы волновой функции было появление суммы, что соответствует неявному предположению, что обе частицы могут равным образом попадать в измерительные устройства для величин $A$ и $B$.

Для двух квантовых состояний нам, следовательно, нужно иметь два бра и два кет, т. е. четыре независимых элемента. Формулы (10) и (11) показывают, что у нас имеются два состояния, занятые с вероятностью единица и две наблюдаемые частицы. По правилам квантовой механики для каждого числа заполнения и для каждой наблюдаемой величины обязательны два элемента бра и кет. Эти элементы согласно формулам (10) и (11) нужны для расчета среднего значения наблюдаемой физической величины.

Теперь предположим, что величины $A$ и $B$ наблюдаются на опыте соответствующими экспериментальными установками - детекторами, причем существует возможность записи измерений каждого детектора и сравнения измерений, относящихся к одному моменту времени. Наблюдения проводятся в двух точках пространства-времени, разделенных пространственноподобным интервалом, что, как уже было сказано, исключает их взаимное влияние.

В результате таких измерений мы получим серии конкретных данных, относящихся к каждому детектору в отдельности. При независимом усреднении показаний детекторов $A$ и $B$ мы получим формулы (10) и (11), причем составленный из них коррелятор $\overline{A B}$ окажется простым произведением средних значений $\bar{A}$ и $\bar{B}$

$$
\overline{A B}_{\text {uncor }}=[\langle k|A| k\rangle\langle l|B| l\rangle+\langle l|A| l\rangle\langle k|B| k\rangle] F_{k} F_{l} .
$$

Это выражение описывает процесс, когда чистые $(k k)$ и $(l l)$ бра + кет пары с вероятностью $F_{k} F_{l}$ попадают в оба детектора. Никакой корреляции между показаниями детекторов в этом случае нет.

Иной результат получится при совместном усреднении показаний детекторов при условии, что усреднять удастся показания детекторов, относящихся к взаимно коррелированным смешанным $(k l)$ и $(l k)$ бра + кет парам. Случайные фазы в таких произведениях показаний 
сократятся, и результат такого усреднения будет соответствовать формуле квантовой механике для коррелятоpa $\overline{A B}=\left\langle\Psi\left(r, r^{\prime}\right)|A B| \Psi\left(r, r^{\prime}\right)\right\rangle$. Этот коррелятор для двух состояний имеет вид

$$
\overline{A B}=[\langle k|A| k\rangle\langle l|B| l\rangle \pm\langle k|A| l\rangle\langle l|B| k\rangle] F_{k} F_{l}+(k \rightleftarrows l) .
$$

Как мы видим, помимо некоррелированного вклада (12) от чистых бра + кет пар в этом корреляторе появится вклад от смешанных бра + кет пар

$$
\overline{A B}_{\text {cor }}=[\langle k|A| l\rangle\langle l|B| k\rangle+\langle l|A| k\rangle\langle k|B| l\rangle] F_{k} F_{l} .
$$

В этом случае с той же вероятностью $F_{k} F_{l}$ в оба детектора попадают две коррелированные по фазе смешанные пары. Их знакопеременный вклад, исчезающий при независимом усреднении в каждом детекторе, становится наблюдаемым при совместном усреднении.

Именно этот вклад обеспечивает наблюдаемую на опыте корреляцию физических величин, разделенных пространственно-подобным интервалом. При отсутствии взаимодействия фазовая когерентность смешанных бра + кет пар может сохраняться на больших временах и расстояниях. Два детектора с корреляционным сигналом сравнения можно считать своеобразным двухчастичным оператором, который описывает взаимодействие, не зависящее от расстояния. Без такого двухчастичного оператора увидеть обменный вклад невозможно.

Напомним еще раз, что в корреляционных опытах с двумя наблюдаемыми частицами мы имеем дело не с двумя, а с четырьмя величинами. Каждая наблюдаемая квантовая частица описывается двумя величинами - бра и кет, которые имеют свои собственные пространственно-временные координаты и эволюционируют независимо.

В опытах по макроскопической квантовой корреляции огромные усилия предпринимаются, чтобы исключить любую реальную или воображаемую связь между детекторами [14-18]. Поскольку обшая причина в прошлом для наблюдаемой корреляции остается неизвестной как сторонникам, так и противникам мгновенного дальнодействия, якобы присущего квантовой механике, проблема обратной связи при измерениях (loopholes) считается крайне важной. Но, как мы видели, такая общая причина в прошлом в виде фазовых соотношений между волновыми функциями участвующих частиц реально сушествует, и, следовательно, вся борьба с воображаемой обратной связью в корреляционных опытах представляется излишней.

Ниже мы рассмотрим примеры проявления квантовой корреляции в некоторых физических эффектах.

\section{4. Кулоновская обменная энергия}

В качестве двухчастичного оператора возьмем кулоновский потенциал, действующий между двумя точками пространства $R=\left|r-r^{\prime}\right|$. Матричный элемент кулоновского потенциала на двухчастичной функции дает энергию взаимодействия квантовых частиц на расстоянии $R$. Он равен сумме двух слагаемых - обычного кулоновского вклада и обменного кулоновского вклада

$$
\begin{aligned}
W= & e^{2} \int_{V} d r d r^{\prime}\left[\frac{\left|\psi_{k}(r)\right|^{2}\left|\psi_{l}\left(r^{\prime}\right)\right|^{2}}{\left|r-r^{\prime}\right|}\right. \\
& \left.-\frac{\psi_{k}^{\dagger}(r) \psi_{l}(r) \psi_{l}^{\dagger}\left(r^{\prime}\right) \psi_{k}\left(r^{\prime}\right)}{\left|r-r^{\prime}\right|}\right] .
\end{aligned}
$$

Первое слагаемое, как мы видим, есть обычное взаимодействие двух зарядов, распределенных в пространстве с заданной плотностью. Плотность заряда в данной точке пропорциональна вероятности обнаружить наблюдаемую частицу в этой точке. Второе слагаемое носит название обменной энергии и обычно объясняется словами: „это квантовый эффект, не имеющий классического аналога“. Знак обменного вклада для фермионов отрицателен, что соответствует запрету Паули для одинаковых квантовых состояний. Для заряженных бозонов он положителен и для одинаковых состояний соответствует сложению амплитуд вероятностей (а не самих вероятностей).

Заметим, что постоянная Планка не входит в это выражение, если ее выписать в явном виде. А кулоновский потенциал явно указывает, что получившаяся энергия связана со взаимодействием электрических зарядов. Выясним, какие это заряды и почему они отличаются от зарядов в первом слагаемом.

Если взять внешний пробный заряд, то он покажет наличие электростатического потенциала, создаваемого распределениями зарядов $e\left|\psi_{k}(\mathbf{r})\right|^{2}$ и $e\left|\psi_{l}(\mathbf{r})\right|^{2}$ :

$$
\Phi(\mathbf{R})=e \int_{V} d \mathbf{r} \frac{\left|\psi_{k}(\mathbf{r})\right|^{2}}{|\mathbf{R}-\mathbf{r}|}+e \int_{V} d \mathbf{r}^{\prime} \frac{\left|\psi_{l}\left(\mathbf{r}^{\prime}\right)\right|^{2}}{\left|\mathbf{R}-\mathbf{r}^{\prime}\right|}
$$

Обменный вклад исчезает из-за ортогональности состояний $k$ и $l$. Выражения (15) и (16) указывают на то, что заряды и поля, ответственные за появление обменного вклада в энергию, не постоянны и должны быть зарядовыми флуктуациями над постоянным фоном. Эти флуктуации, как видно из формулы (15), описываются произведениями бра и кет функций с разными квантовыми индексами $\psi_{k}^{\dagger}(r) \psi_{l}(r)$ и $\psi_{l}^{\dagger}\left(r^{\prime}\right) \psi_{k}\left(r^{\prime}\right)$. В среднем такие флуктуации равны нулю, но возникают синхронно и успевают взаимодействовать и дать вклад в энергию. Быстрый кулоновский потенциал в данном случае служит индикатором наличия корреляции между возникающими зарядовыми флуктуациями.

В опытах по макроскопической квантовой корреляции данные детекторов в один момент времени записываются и потом сравниваются. Кулоновский потенциал реагирует на одновременное появление зарядов в двух точках пространства, заменяя тем самым сложную систему записи и сравнения данных детекторов. 


\section{5. Эффект Ханбери Брауна-Твисса}

Квантовая корреляция на макроскопических расстояниях наблюдалась впервые как корреляция интенсивностей электромагнитных волн, испущенных удаленными источниками [19]. Для описания такой корреляции подходит основная формула (13), где надо взять волновые функции в виде плоских волн, а операторы $A$ и $B$ будут так называемыми операторами положения дельта-функциями $\delta\left(r-r_{1}\right)$ и $\delta\left(r^{\prime}-r_{2}\right)$ в двух точках пространства. Для коррелятора в этом случае получим

$$
\overline{A B}=\left[1 \pm \cos \left(p-p^{\prime}\right) R\right] F_{p} F_{p^{\prime}},
$$

где $r_{1}-r_{2}=R$. Это выражение описывает также бунчировку бозонов и антибунчировку фермионов в многочастичных квантовых системах. Например, в опытах, где атомы гелия-3 (фермионы) или гелия-4 (бозоны) падают на плоскость, получается как раз такая картина. Первый (единичный) вклад в формуле (17) - это вклад чистых бра + кет пар $(p p)$ и $\left(p^{\prime} p^{\prime}\right)$. Второй вклад обеспечивают смешанные $\left(p p^{\prime}\right)$ и $\left(p^{\prime} p\right)$ бра + кет пары.

Эффект Ханбери Брауна-Твисса используется в физике элементарных частиц для измерения корреляции потоков частиц при ядерных реакциях. Заметим, что его объяснение в литературе тождественностью квантовых частиц эквивалентно, по сушеству, словам „это квантовый эффект".

\section{6. Корреляция двух спинов на расстоянии}

Ввиду большого внимания к корреляции спинов на макроскопических расстояниях, рассмотрим спиновую корреляцию более подробно. В этом случае в основной формуле (13) операторы наблюдаемых спинов в детекторах $A$ и $B$ надо взять в виде скалярных произведений вектора $\sigma$ из трех матриц Паули и единичных векторов $n=a$ и $n=b$, задающих направления измерений. Волновые функции $k\rangle \equiv|\uparrow\rangle$ и $|l\rangle \equiv \downarrow\rangle$ теперь будут собственными функциями оператора $\sigma_{z}$ с собственными значениями $s_{\uparrow}=1$ и $s_{\downarrow}=-1$. Удобно использовать полярные координаты для углов наблюдения. При этом $S \equiv(n, \sigma)=\sin \theta \cos \varphi \sigma_{x}+\sin \theta \sin \varphi \sigma_{y}+\cos \theta \sigma_{z}$.

Для большей ясности рассмотрим вклады от чистых и смешанных бра-кет пар по отдельности. Для чистых пар только $\sigma_{z}$ дает вклад в матричный элемент оператора $S$, и мы имеем

$$
\begin{gathered}
\langle\uparrow|S| \uparrow\rangle=\cos \theta\left\langle\uparrow\left|\sigma_{z}\right| \uparrow\right\rangle=\cos \theta, \\
\langle\downarrow|S| \downarrow\rangle=\cos \theta\left\langle\downarrow\left|\sigma_{z}\right| \downarrow\right\rangle=-\cos \theta .
\end{gathered}
$$

Для смешанных бра-кет пар получаем:

$$
\begin{aligned}
\langle\uparrow|S| \downarrow\rangle= & \cos \varphi \sin \theta\left\langle\uparrow\left|\sigma_{x}\right| \downarrow\right\rangle \\
& +\sin \varphi \sin \theta\left\langle\uparrow\left|\sigma_{y}\right| \downarrow\right\rangle=e^{i \varphi} \sin \theta, \\
\langle\downarrow|S| \uparrow\rangle= & \cos \varphi \sin \theta\left\langle\downarrow\left|\sigma_{x}\right| \uparrow\right\rangle \\
+ & \sin \varphi \sin \theta\left\langle\downarrow\left|\sigma_{y}\right| \uparrow\right\rangle=e^{-i \varphi} \sin \theta .
\end{aligned}
$$

Средние значения наблюдаемых спинов в двух детекторах равны

$$
\begin{gathered}
\bar{A}=\langle\uparrow|A| \uparrow\rangle F_{\uparrow}+\langle\downarrow|A| \downarrow\rangle F_{\downarrow}=\cos \theta_{a}\left(F_{\uparrow}-F_{\downarrow}\right), \\
\bar{B}=\langle\uparrow|B| \uparrow\rangle F_{\uparrow}+\langle\downarrow|B| \downarrow\rangle F_{\downarrow}=\cos \theta_{b}\left(F_{\uparrow}-F_{\downarrow} .\right.
\end{gathered}
$$

Заметим, что для $F_{\uparrow}=F_{\downarrow}$ средние значения спинов $\bar{A}$ и $\bar{B}$ равны нулю независимо от выбранного направления измерения. Для коррелятора $\overline{A B}$, как и раньше, имеем два вклада. Первый равен

$$
(\overline{A B})_{\text {pure }}=-\cos \theta_{a} \cos \theta_{b} 2 F_{\uparrow} F_{\downarrow} .
$$

За второй вклад ответственны смешанные бра-кет пары, для которых мы имеем:

$$
(\overline{A B})_{\text {mixed }}=-\cos \left(\varphi_{a}-\varphi_{b}\right) \sin \theta_{a} \sin \theta_{b} 2 F_{\uparrow} F_{\downarrow} .
$$

Сумма этих вкладов равна

$$
\overline{A B}=-\cos \gamma 2 F_{\uparrow} F_{\downarrow},
$$

где

$$
\cos \gamma=\cos \theta_{a} \cos \theta_{b}+\cos \left(\varphi_{a}-\varphi_{b}\right) \sin \theta_{a} \sin \theta_{b} .
$$

Таким образом, коррелятор спинов зависит только от угла между двумя измерениями поляризации и не зависит от исходной ориентации поляризации. В этом отношении он подобен равному нулю среднему спину при равенстве чисел заполнения состояний с противоположными направлениями спинов в (20).

В литературе коррелятор $\overline{A B}$ расчитывается как матричный элемент $\langle\Psi|A B| \Psi\rangle$ от синглетной волновой функции $\Psi$

$$
\Psi=\left|\uparrow_{a}\right\rangle\left|\downarrow_{b}\right\rangle-\left|\uparrow_{b}\right\rangle\left|\downarrow_{a}\right\rangle .
$$

Отсюда

$$
\begin{gathered}
\overline{A B}=\langle\Psi|A B| \Psi\rangle, \\
\bar{A}=\langle\Psi|A| \Psi\rangle=0, \quad \bar{B}=\langle\Psi|B| \Psi\rangle=0 .
\end{gathered}
$$

Получающееся выражение для коррелятора $\overline{A B}$ совпадает с нашим выражением (21) при условии $F_{\uparrow}=F_{\downarrow}$ и $F_{\uparrow} F_{\downarrow}=1$. Важно подчеркнуть, что потоки спинов от независимых источников дают тот же самый результат, как и поток спинов, описываемых синглетной спиновой волновой функцией.

Нужно помнить, что корреляция носит статистический характер, и для ее наблюдения нужны серии измерений. Неучет этого важного обстоятельства часто приводит к неоправданным и неверным выводам из формул (21)-(23). Так, например, среднее значение спина в каждом детекторе, найденное с помощью синглетной волновой функции, равно нулю, независимо от выбранного направления измерения. Очень часто это обстоятельство приводят как аргумент в пользу неких особых свойств синглетного состояния. Утверждается, что в этом состоянии между двумя спинами существует 
таинственная связь, так что при измерении одного спина второй спин немедленно принимает противоположное направление, причем это происходит независимо от расстояния между ними.

Если взглянуть на формулу (13), то становится очевидным, что никакими особыми свойствами синглетное состояние не обладает.

Действительно, поток поляризованых по оси $z$ спинов, измеряемый под углом $\theta$, дает среднее значение спина в соответствии с законом Малюса

$$
\bar{A}=\langle\uparrow|A| \uparrow\rangle F_{\uparrow}=\cos \theta F_{\uparrow} .
$$

Это среднее значение реализуется в виде случайного набора единиц разного знака, которые появляются с вероятностями, соответствующими углу наблюдения

$$
\begin{aligned}
\cos \theta & =(+1) \cos ^{2} \theta / 2+(-1) \sin ^{2} \theta / 2 \\
& =\cos ^{2} \theta / 2-\sin ^{2} \theta / 2 .
\end{aligned}
$$

Если в потоке присутствуют спины противоположных знаков, то среднее значение будет пропорционально также разности вероятностей их появления

$$
\bar{A}=\langle\uparrow|A| \uparrow\rangle F_{\uparrow}+\langle\downarrow|A| \downarrow\rangle F_{\downarrow}=\cos \theta\left(F_{\uparrow}-F_{\downarrow}\right) .
$$

В синглетном состоянии таких спинов поровну, что и обеспечивает ноль в среднем по любому направлению. Как мы видим, предполагать наличие между спинами магической взаимной связи, распространенной на всю Вселенную, совсем не обязательно.

Заметим, что при $\theta=\pi / 2$ детектор также покажет средний ноль согласно (20). Это случится безотносительно к числу положительных или отрицательных спинов. В этом случае для $N$ положительных спинов получится в среднем $N / 2$ плюс-единиц и равное количество минус-единиц. Для $M$ отрицательных спинов точно также получится $M / 2$ плюс-единиц и такое же количество минус-единиц.

Покажем теперь как неравенство Белла [17] нарушается вкладом смешанных взаимно коррелированных бра-кет пар. Нарушение неравенства на опыте служит доказательством правильности формул квантовой механики для коррелятора спинов (21). В типичном эксперименте (см., например, $[12,13]$ ) в детекторы $A$ и $B$ попадают потоки противоположно поляризованных спинов равной интенсивности. Над этими потоками в детекторах одновременно делаются серии наблюдений для четырех комбинаций из двух направлений измерения $\mathbf{a}$ и $\mathbf{a}^{\prime}$ в детекторе $A$ и двух направлений $\mathbf{b}$ и $\mathbf{b}^{\prime}$ в детекторе $B$. Из сделанных наблюдений составляются усредненные комбинации показаний детекторов

$$
D \equiv\left|\overline{A B}+\overline{A B^{\prime}}+\overline{A^{\prime} B}-\overline{A^{\prime} B^{\prime}}\right| .
$$

Серии измерений в каждом детекторе представляют собой набор положительных и отрицательных единиц.
Если (в противоречии с принципами квантовой механики!) предположить, что значения спинов существуют до измерения и всегда равны \pm 1 , то $D \leq 2$. При этом согласно Беллу любая предварительная корреляция значений спинов существующих до измерения не может нарушить неравенство. Это утверждение носит название теоремы Белла.

Однако при подстановке в $D$ квантово-механического выражения для коррелятора (23) и при определенном выборе направлений измерений неравенство Белла нарушается. Так, пусть векторы $\mathbf{a}$ и $\mathbf{b}$ паралельны оси $z$, так что $\theta_{a}=\theta_{b}=0$, а векторы $\mathbf{a}^{\prime}$ и $\mathbf{b}^{\prime}$ взаимно ортогональны и идут под углом $45^{\circ}$ к оси $z$. При этом $\theta_{a^{\prime}}=-\theta_{b^{\prime}}=\pi / 4$.

Для данного случая имеем

$$
\begin{aligned}
\mid \cos \gamma_{a b} & +\cos \gamma_{a b^{\prime}}+\cos \gamma_{a^{\prime} b}-\cos \gamma_{a^{\prime} b^{\prime}} \mid \\
& =|1+1 / \sqrt{2}+1 / \sqrt{2}-0|=1+\sqrt{2}>2 .
\end{aligned}
$$

В этой сумме первые три слагаемых идут от чистых некоррелированных бра-кет пар. Действительно, для выбранной геометрии опыта $\sin \theta_{a}=\sin \theta_{b}=0$ и вклад смешанных пар отсутствует. Он присутствует только в четвертом слагаемом (27), где некоррелированные и коррелированные вклады в (20) сокращают друг друга: $\gamma_{a^{\prime} b^{\prime}}=\cos (\pi / 2)=0$. Если исключить вклад смешанных пар, то оставшаяся часть будет удовлетворять неравенству Белла: $|1+1 / \sqrt{2}+1 / \sqrt{2}-1 / 2|=1 / 2+\sqrt{2}<2$.

Как мы видим, именно знакопеременный вклад смешанных бра-кет пар в показания каждого детектора, который проявляется только при их совместном усреднении, но не виден при усреднении показаний каждого детектора в отдельности, оказывается причиной нарушения неравенства Белла. Обязательным условием при этом является усреднение именно взаимно коррелированных по фазе смешанных пар. Отметим еще раз, что эти коррелированные пары возникают при обмене бра или кет, взятых из двух чисел заполнения, где фазы бра и кет равны по величине и противоположны по знаку.

При доказательстве теоремы Белла существенно используется положительность вероятности для значений спинов. Смешанные состояния не описываются такой вероятностью и не участвуют в нормировке чисел заполнения на полное число частиц. Именно поэтому их вклад не учитываются при выводе неравенства Белла.

\section{7. Общая причина в прошлом - источник корреляции}

Итак, существование чисел заполнения для бра-кет пар и возможность обмена между ними с образованием новых взаимно коррелированных бра-кет пар является скрытой „общей причиной в прошлом“ для наблюдаемой корреляции физических величин в один момент времени. 
Смешанные бра-кет пары с равными и противоположными фазами можно считать теми самыми запутанными состояниями, о которых так много пишется в литературе. Несмотря на повсеместное употребление этого термина, мы не нашли ясного указания, в чем же, собственно, заключается эта самая запутанность.

При отсутствии взаимодействия бра и кет фазы могут сохраняться на больших временах и больших расстояниях, что и наблюдается в опытах по макроскопической корреляции.

Во избежание недоразумений следует подчеркнуть, что коррелированные по фазе пары существуют независимо друг от друга и любое действие над одной парой не влияет другую. Так что измерения в разных детекторах никоим образом не могут влиять друг на друга, как это часто утверждается в литературе по спиновой $\operatorname{EPR}(\mathrm{B})$-корреляции. Несмотря на существование коррелированных по фазе бра-кет состояний и их вклад в наблюдаемую на опыте корреляцию физических величин на макроскопических расстояниях, квантовая механика остается локальной. Вопреки мнению сторонников нелокальности нарушение неравенства Белла лишь подтверждает справедливость формул квантовой механики, но не противоречит ее локальности.

\section{8. Обменная корреляция и флуктуации}

Как известно, в равновесии отклик и флуктуации связаны флуктуационно-диссипационной теоремой (ФДТ). Эта теорема основывается на двух положениях. Во-первых, созданное внешним возмушением отклонение от равновесия и отклонение, возникшее спонтанно релаксируют к равновесию одинаковым образом. Это постулат Онзагера. Второе положение - отсутствие корреляций между числами заполнения состояний в один момент времени, т.е. имеет место только автокорреляция.

Для квантовых частиц ФДТ выводится с помошью множителя Гиббса в выражении для усреднения наблюдаемой величины по ансамблю, а не при помощи равновесных функций распределения Бозе и Ферми. Сравнение отклика и флуктуаций с учетом постулата Онзагера приводит к сотношению между ними, которое определяется их начальными значениями, в которые функции распределения входят по-разному. Для пары фермионов или бозонов в состояниях $k$ и $l$ имеем

$$
\begin{gathered}
R_{k l}=F_{k}\left(1 \mp F_{l}\right)-F_{l}\left(1 \mp F_{k}\right)=F_{k} F_{l}\left(1 / F_{l}-1 / F_{k}\right), \\
\left.\Phi_{k l}=F_{k}\left(1 \mp F_{l}\right)+F_{l}\right)\left(1 \mp F_{k}\right)=F_{k} F_{l}\left(1 / F_{l}+1 / F_{k} \pm 2\right) .
\end{gathered}
$$

В равновесии $1 / F(\epsilon)=e^{(\epsilon-\mu) / T} \mp 1$. При условии $\omega=\epsilon_{k}-\epsilon_{l}$ получаем:

$$
K_{k l}=\frac{R_{k l}}{\Phi_{k l}}=\frac{1 / F_{l}-1 / F_{k}}{1 / F_{l}+1 / F_{k} \pm 2}=\frac{e^{\epsilon_{k} / T}-e^{\epsilon_{l} / T}}{e^{\epsilon_{k} / T}+e^{\epsilon_{l} / T}}=\text { th } \frac{\omega}{2 T} .
$$

Это равновесное термодинамическое соотношение между откликом и флуктуациями получается для статистики Ферми или Бозе только при учете обменного корреляционного вклада. Наоборот, для классических частиц, подчиняющихся статистике Больцмана, обмен по физике дела невозможен и корреляционный вклад исключается. Заметим, что при обычном формальном выводе флуктуационно-диссипационной теоремы роль обменной корреляции для квантовых частиц не видна.

Для квазиклассических равновесных флуктуаций в газе фермионов и бозонов для существования ФДТ требуется равенство

$$
N \frac{\partial F_{p}}{\partial N}=F_{p}\left(1 \mp F_{p}\right)
$$

Левая часть этого равенства возникает в задаче об отклике, правая часть в задаче о флуктуациях и описывает обменную корреляцию для одинаковых квантовых состояний. Если определить равновесное состояние как состояние, где существует ФДТ, то формула определяет форму функции распределения такого равновесного состояния. Заметим, что для произвольной функции распределения, равенство (32) заведомо не выполняется, что служит явным признаком нарушения связи между откликом и флуктуациями в неравновесном газе фермионов и бозонов. В таких газах обменный корреляционный механизм при рассеянии на фононах и примесях может конкурировать с корреляционным механизмом от парных столкновений.

\section{9. Микроскопические обменные эфффекты}

Как мы видели, обменная корреляция в многоэлектронной системе, как и другие микроскопические корреляционные эффекты, имеет одну и ту же физическую природу с макроскопической корреляцией на макроскопических расстояниях. Сходство между этими эффектами было замечено и время от времени отражалось в физической литературе, однако почти всегда как проявление нелокальности и мгновенной связи между квантовыми состояниями (см., например, [20-22]). Как было показано выше, в действительности наблюдаемая корреляция объясняется „общей причиной в прошлом“ - действием обменного корреляционного механизма, основанного на существовании чисел заполнения с одинаковыми фазами для бра и кет волновых функций - решений уравнения Шредингера. Не таинственное мгновенное дальнодействие, а присутствие в многочастичной системе когерентных коррелированных по фазе состояний является источником наблюдаемой на опыте микроскопической корреляции наблюдаемых физических величин. Сравнение макроскопической и микроскопической корреляции позволяет лучше понять физику квантовой корреляции в том и другом случае. Обменная квантовая корреляция может играть важную роль в образовании квантового 
конденсата, для чего нужна многочастичная корреляция из цепочек коррелированных смешанных состояний вида

$$
\left\langle\psi_{1}|A| \psi_{2}\right\rangle\left\langle\psi_{2}|B| \psi_{3}\right\rangle\left\langle\psi_{3}|C| \psi_{4}\right\rangle\left\langle\psi_{4}|D| \psi_{5}\right\rangle \ldots \text {. }
$$

Для одинаковых волновых функций весь конденсат описывается парой макроскопических бра и кет функций, что и определяет своеобразие таких физических образований.

\section{0. Заключение}

Как мы видели квантовые корреляционные эффекты как на микроскопических так и на макроскопических расстояниях имеют одну и ту же физическую природу. Для их объяснения и интерпретации вполне достаточно общепринятых положений и формул квантовой механики. Какая-то новая интерпретация квантовой механики, отличная от стандартной копенгагенской интерпретации, нам представляется излишней.

Нужно только хорошо помнить, что состояние физической системы задается не одной только волновой функцией, а набором всех возможных наблюдаемых физических величин в этом состоянии. Линейность уравнения Шредингера и билинейность формул для наблюдаемых физических величин естественным образом приводит к возникновению двух типов физических состояний - чистых и смешанных квантовых состояний. Чистые квантовые состояния описываются парой волновых функций $($ ба + кет) с одинаковым квантовым индексом и соответствуют диагональным матричным элементам наблюдаемой физической величины. Эти элементы не зависят от времени и от начальных фаз волновых функций и определяют постоянный фон наблюдаемых. Смешанные квантовые состояния, которые описываются бра + кет функциями с различными квантовыми индексами, дают значения наблюдаемых, зависящие от времени и от начальных фаз волновых функций. Вклад от этих смешанных состояний можно рассматривать как флуктуации над фоном от чистых состояний. При наличии занятых состояний с числами заполнения флуктуации возникают когерентно в разных точках пространства, обеспечивая пространственную корреляцию значений физических величин в один момент времени. Наблюдаемая на макроскопических расстояниях квантовая корреляция как раз и есть проявление таких когерентных флуктуаций, а вовсе не какого-то мгновенного взаимодействия на расстоянии, якобы присущего квантовой механике.

Следует подчеркнуть, что только на языке бра + кет пар различие между двумя типами состояний квантовой системы делается достаточно ясным и очевидным. Наоборот, если рассматривать только волновые функции, это различие смазывается, а физическая картина становится неясной. Как пример подобной неясности, можно привести воспоминание Гейзенберга о многодневной утомительной дискуссии между Бором и Шредингером о стационарных орбитах в атоме и „квантовых скачках“ между этими орбитами, где оба использовали только волновые функции и не договорились [23]. На языке бра + кет предмет спора исчезает, так как чистые бра + кет пары сопоставляются стационарным орбитам Бора, а естественно возникающие при переходах между ними смешанные бра + кет пары описывают „квантовые скачки“, существование которых отрицал Шредингер.

Многие другие трудные и неясные вопросы интерпретации квантово-механических формул становятся гораздо более ясными и понятными на бра + кет языке.

\section{Список литературы}

[1] S.V. Gantsevich, V.L. Gurevich. Theor. Phys. (Italy) 2, 2, 63 (2017).

[2] A. Einstein, B. Podolsky, N. Rosen. Phys. Rev. 47, 777 (1935).

[3] N. Brunner, D. Cavalcanti, S. Pironio, V. Scarani, S. Wehner. Rev. Mod. Phys. 86, 419 (2014).

[4] M.D. Reid, P.D. Drummond, W.P. Bowen, E.G. Cavalcanti, P.K. Lam, H.A. Bachor, V.L. Andersen, G. Leuchs. Rev. Mod. Phys. 81, 1727 (2009).

[5] L. Amico, R. Fazio, A. Osterloh, V. Vedral. Rev. Mod. Phys. 80, 518 (2008).

[6] W.H. Zurek. Rev. Mod. Phys. 75, 715 (2003).

[7] A. Zeilinger. Rev. Mod. Phys. 71, S288 (1999).

[8] Д.Н. Клышко. УФН 154, 1187 (1988); 158, 327 (1989).

[9] Д.Н. Клышко. УФН 164, 133 (1994); 168, 975 (1998).

[10] Б.И. Спасский, А.В. Московский. УФН 142, 4, 599 (1984).

[11] А.А. Гриб. УФН 142, 4, 619 (1984).

[12] Б.Б. Кадомцев. УФН 168, 9, 975 (2004).

[13] Б.Б. Кадомцев. Динамика и информация. Наука, М. (2004). C. 118,315 .

[14] B. Hensen, H. Bernien, A.E. Dréau, A. Reiserer, N. Kalb, M.S. Blok, J. Ruitenberg, R.F.L. Vermeulen, R.N. Schouten, C. Abelian, W. Amaya, V. Pruneri, M.W. Mitchell, M. Markham, D.J. Twitchen, D. Elkouss, S. Wehner, T.H. Taminiau, R. Hanson. Nature 526, 682 (2015).

[15] H. Wiseman. Nature 526, 649 (2015).

[16] J. Handsteiner, A.S. Friedman, D. Rauch, J. Gallicchio, B. Liu, H. Hosp, J. Kofler, D. Bricher, M. Fink, C. Leung, A. Mark, H.T. Nguyen, I. Sanders, F. Steinlechner, R. Ursin, S. Wengerowsky, A.H. Guth, D.I. Kaiser, T. Scheidl, A. Zelinger. Phys. Rev. Lett. 118, 060401 (2017).

[17] J.S. Bell. Physics 1, 195 (1964).

[18] D. Bohm, Y. Aharonov. Phys. Rev. 108, 1070 (1957).

[19] R.H. Brown, R.Q. Twiss. Nature (London) 177, 27 (1956); 178, 1046 (1956).

[20] J.M. Raimond, M. Brune, S. Haroche. Rev. Mod. Phys. 73, 565 (2001).

[21] P. Samuelsson, E.V. Sukhorukov, M. Buttiker. Phys. Rev. Lett. 92, 026805 (2004).

[22] P. Samuelsson, E.V. Sukhorukov, M. Buttiker. Phys. Rev. Lett. 84, 1035 (2000).

[23] W. Heisenberg. В сб. Нильс Бор и развитие физики. ИНЛ, M. (1958). 